\title{
Diffusion-Controlled Growth of Oxygen Bubble Evolved from Nanorod-Array $\mathrm{TiO}_{2}$ Photoelectrode
}

\author{
Xiaowei Hu, Yechun Wang, Liejin Guo, and Zhenshan Cao \\ State Key Laboratory of Multiphase Flow in Power Engineering, International Research Center for Renewable Energy, \\ Xian Jiaotong University, Shaanxi 710049, China \\ Correspondence should be addressed to Xiaowei Hu; hu-xiaowei@mail.xjtu.edu.cn and Liejin Guo; lj-guo@mail.xjtu.edu.cn
}

Received 28 March 2014; Accepted 8 May 2014; Published 25 May 2014

Academic Editor: Haimin Zhang

Copyright (C) 2014 Xiaowei Hu et al. This is an open access article distributed under the Creative Commons Attribution License, which permits unrestricted use, distribution, and reproduction in any medium, provided the original work is properly cited.

Nanorod-array structure gains its popularity in photoelectrode design for water splitting. However, the structure's effects on solid-liquid interface interaction and reaction product transportation still remain unsolved. Gas bubble generally evolved from photoelectrodes, which provides a starting point for the problem-solving. Based on this, investigations on the gas-evolving photoelectrode are carried out in this paper. By experimental studies of wettability on the photoelectrode nanorod-array surface and oxygen bubble growth from anode, we analyzed the interaction affecting the gas-solid-liquid contact behaviors and product transportation mechanism, which is controlled by diffusion due to the concentration gradient of dissolved gases in the aqueous electrolyte and the microconvection caused by the bubble interface movement. In the end, based on the bubble growth characteristics of $R_{\mathrm{B}}(\mathrm{t}) \sim t^{0.5}$ in the experiment, a model describing the product transport mechanism was presented.

\section{Introduction}

Hydrogen production by photoelectrochemical water splitting is a promising source for carbon-free energy [1-3]. In order to increase the energy conversion efficiency, a lot of research on transfer process enhancement of photo-electron has been carried out with great progress by photoelectrode surface modification with special micro-nano structure [47]. But for this kind of heterogeneous photocatalytic or photochemical conversion system, many issues concerning interface of such micro-nano structure semiconductor and liquid still remain unsolved, such as the nature of the active sites, mechanism for heterogeneous photocatalytic reaction of water splitting, and effects of interfacial interaction during reaction product transfer.

Much research has been focused on photon-electron transfer movement and enhancement from solid inside to the solid surface, but the principle how the electrons transferring to the surface interact with the water is underinvestigated. Actually, solid surface with roughness or special structures can highly influence the interaction between water and solid surface which is indicated by the parameter of contact angle [8] and will highly influence the surface photocatalytic reaction as follows:

$$
\begin{gathered}
\mathrm{H}^{+}+e^{-} \longrightarrow \frac{1}{2} \mathrm{H}_{2} \\
\mathrm{O}^{2-}+2 h^{+} \longrightarrow \frac{1}{2} \mathrm{O}_{2} .
\end{gathered}
$$

On the other hand, hydrogen/oxygen bubble evolution from photoelectrode is a typical interfacial phenomenon controlled by the interfacial interactions and mass-transfer process in the three-phase zone where gas bubble, electrolyte, and electrode surface contact each other, leading to requirement for understanding the bubble evolution process during photocatalysis especially for photoelectrode with micronano structure surface. Analogy to water electrolysis, it is believed the bubble evolution process includes the generation of the dissolved gas in molecular form by electrochemical reactions, the accumulation of the dissolved gas molecules around the electrode surface to form the supersaturation layer, bubble nucleation, growth, and detachment on surface active sites. For the bubble growth process, the existence 


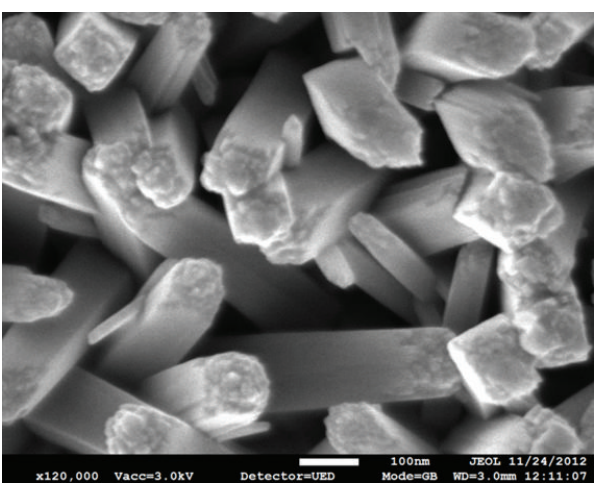

(a)

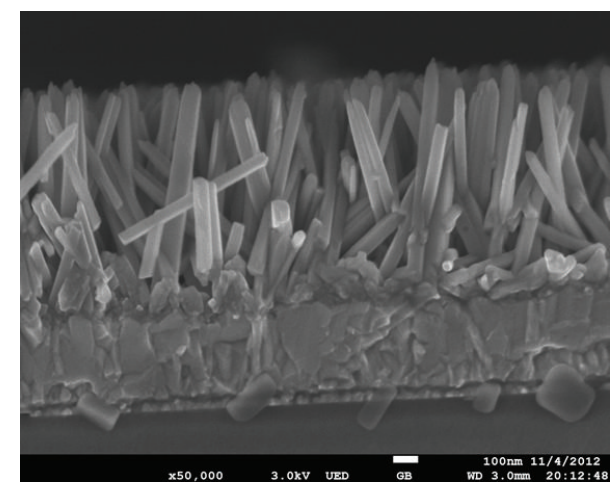

(b)

Figure 1: Nanorod-array structure $\mathrm{TiO}_{2}$ film.

of two characteristic regimes for the increase of the bubble radius $R_{\mathrm{B}}(t)$ on time could be expected. In the first stage, which is difficult to observe, a fast inertia-controlled growth takes place. The resulting reduction of the gas available amount leads to the second regime, diffusion-controlled growth according to $R_{\mathrm{B}}(t) \sim t^{x}$. Generally, the $x$ is considered to be 0.5 as described by Scriven's theory [9]. But if the ratio between photoelectrode size and bubble diameter is small enough to make the gas produced probably directly inject into the bubble, by virtue of the steep concentration gradients at the foot of the bubble, the $x$ is equal to 0.3 $[10,11]$. During these two growth regimes, the effects of diffusion caused by the presence of the supersaturation layer and microconvection due to bubble play an important role, which is owed to the concentration gradient of dissolved gases in the aqueous electrolyte called as single-phase free convection, and interface movement between gas and liquid on electrode surface once the bubble is nucleated, respectively $[12,13]$.

Although much progress has been made on the bubble growth, effects of the solid surface structure are still to be understood. Wei et al. investigated the bubble behavior on micro-pin-fined surface and considered a large capillary force leading to easy bubble detachment [14]. Yang et al. studied the bubble-solid interaction indicating the importance of electrolyte concentration and DLVO surface forces [15]. The interaction between the micro-nano structure surface with electrolyte and gas-liquid interface should be investigated more deeply.

In this paper, to investigate nanostructure effects on bubble behavior evolved from nanoscale structure photoelectrode, a study on nanorod-array structure surface characteristics and single oxygen bubble growth dynamics is carried out.

\section{Experimental}

$\mathrm{TiO}_{2}$ film modified by nanorod-array was used as the anode in the investigation, and its SEM shows in Figure 1 with rod height of about $870 \mathrm{~nm}$ and rod diameter of nearly $120 \mathrm{~nm}$.
The optical contact angle meter model SL200 was applied to study the wettability of its surface.

The experiments on bubble evolution were conducted in an electrolytic cell, as shown in Figure 2. $\mathrm{Na}_{2} \mathrm{SO}_{4}$ in deionized water with different concentrations of $0.495,0.354,0.261$, 0.206 , and $0.170 \mathrm{~mol} / \mathrm{L}$ was used as the electrolyte. Pt was used as a cathode. The bubble evolution process was observed by the microscope camera system. The image intense CCD whose resolution is $1376 * 1040$ and objective lens is $5 x / 0.15$ was applied. All the experiments were conducted under the conditions of ambient temperature $25^{\circ} \mathrm{C}$ and ambient pressure $1 \mathrm{~atm}$.

\section{Results and Discussion}

3.1. Wettability of Nanorod-Array $\mathrm{TiO}_{2}$ Film. In order to investigate the interaction, especially wettability between nanorod-array film and water, we carry out the experiment on contact angle firstly. Figure 3 shows measurement results of about $80^{\circ} \pm 1^{\circ}$, indicating a general hydrophilic ability.

\subsection{Oxygen Bubble Behavior Evolved from Nanorod-Array} $\mathrm{TiO}_{2}$ Film. Figure 4 shows the general characteristics of bubble growth. Compared to bubble growth during boiling and common electrolysis, bubble evolved from nanorodarray surface stays longer and smaller, which is determined by the forces acting on them. In our experiment, the proper effects include gravity, gas-liquid interfacial surface tension, marangoni force, electric force (with the possibility of gasliquid interface being charged), and interfacial interaction with elastic materials. Among those, the most possible and important effects to prevent the bubble departure and keep bubble staying are marangoni force due to nonhomogeneous gas adsorption at the interface and the capillary force caused by contact behavior with nanorod-array surface structure.

Besides the dynamics aspect, we are much concerned about the mechanism driving the bubble growth. We investigate the relationship of $R_{\mathrm{B}}(t) \sim t^{x}$, as show in Figure 5 . It indicates the $R_{\mathrm{B}}(t)^{2}$ is linear to the time, which is to say $x=0.5$ for equation $R_{\mathrm{B}}(t) \sim t^{x}$. 


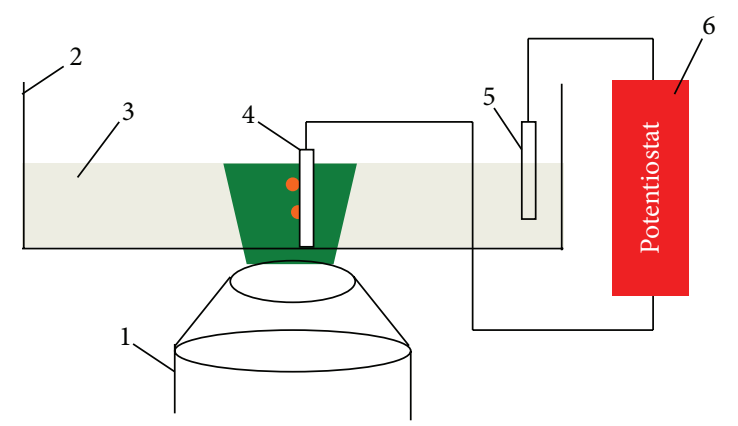
(1) Camera system
(4) $\mathrm{TiO}_{2}$ film with nanorod-array
(2) Electrolytic cell
(5) Pt electrode
(3) Electrolyte
(6) Voltage-stabilized source

FIGURE 2: Schematic of the experiment setup.

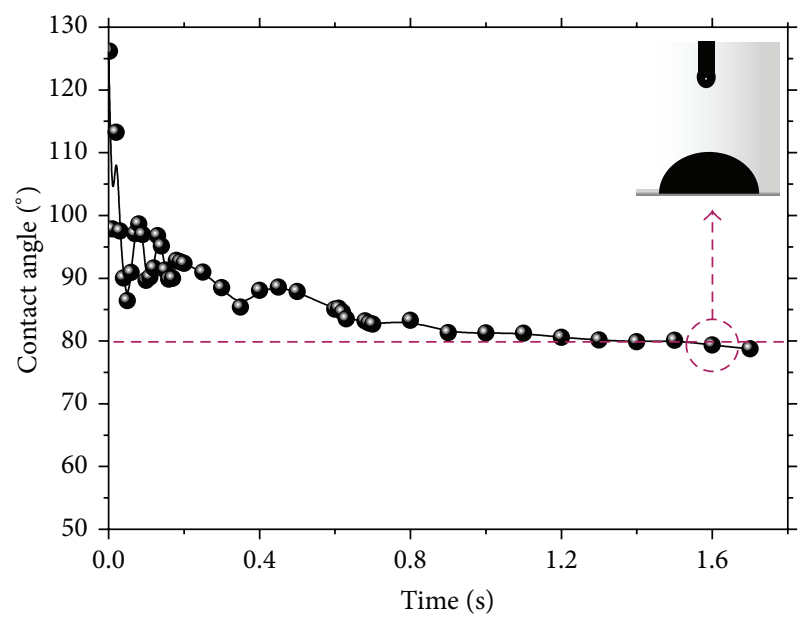

Figure 3: Contact angle measurement results of nanorod-array $\mathrm{TiO}_{2}$ film and water.

3.3. Oxygen Bubble Growth Model from Nanorod-Array $\mathrm{TiO}_{2}$ Film. The bubble growth is driven by the concentration difference between the electrolyte bulk and the bubble interface, which is an analogy to the bubble growth during boiling. But the acquisition of the concentration data, key to electrolysis, is much difficult than that of the temperature data for boiling. So the modelling of bubble growth driven by the concentration difference is less developed than that driven by the temperature difference.

From the above analysis, we obtained $R_{\mathrm{B}}(t) \sim t^{0.5}$ relationship, which is first growth mechanism showed in Figure 6. According to Vogt's theory [12, 13], effects on bubble growth in one circle, meaning the process of bubble departure and movement excluded, include two parts, which are the diffusion due to the concentration gradient of dissolved gases in the aqueous electrolyte and the microconvection caused by the bubble interface movement.

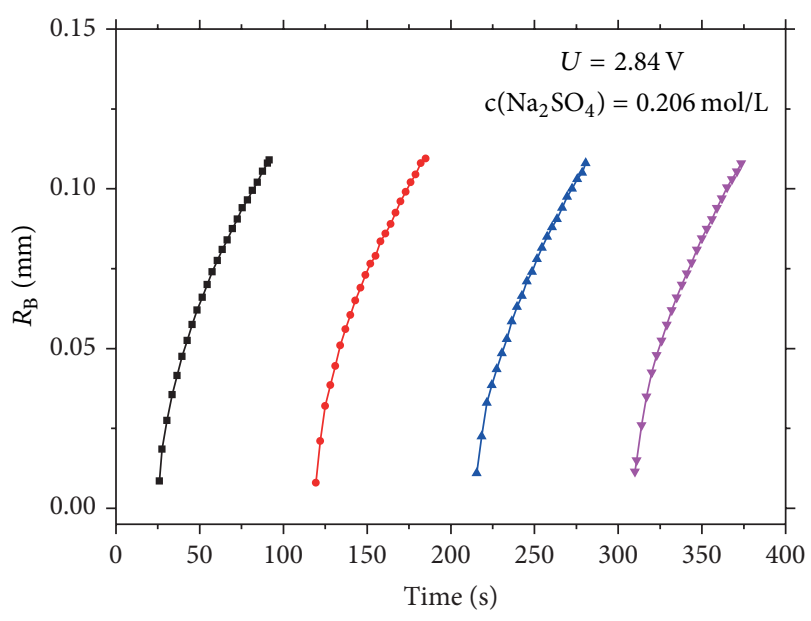

Figure 4: Bubble growth characteristics under $U=2.84 \mathrm{~V}$ and $\mathrm{c}\left(\mathrm{Na}_{2} \mathrm{SO}_{4}\right)=0.206 \mathrm{~mol} / \mathrm{L}$ (colorful lines stand for different growth periods).

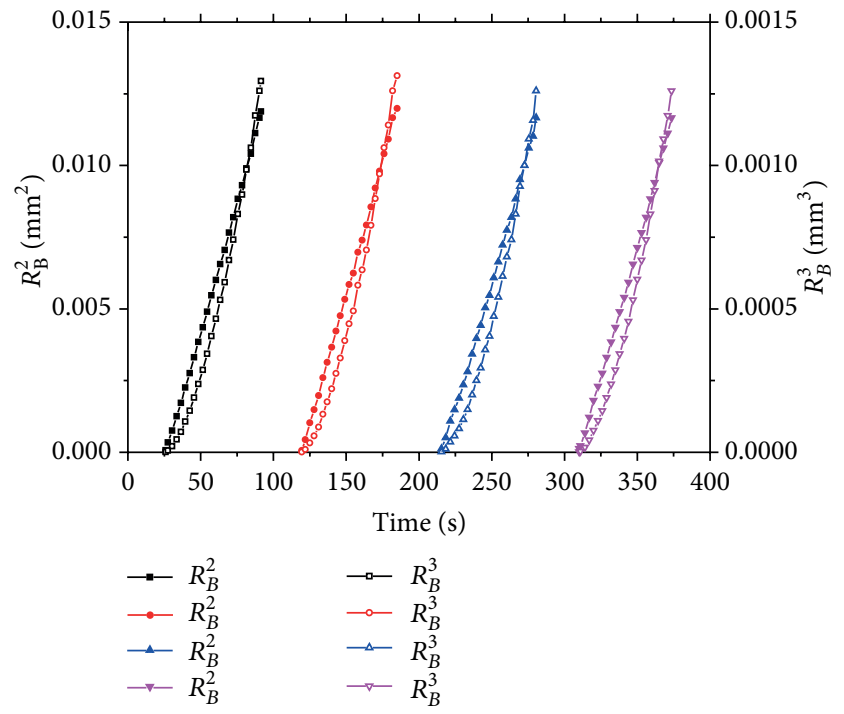

FIGURE 5: The relationship between bubble radius and time under $U=2.84 \mathrm{~V}$ and $\mathrm{c}\left(\mathrm{Na}_{2} \mathrm{SO}_{4}\right)=0.206 \mathrm{~mol} / \mathrm{L}$ (colorful lines stand for different growth periods).

For the diffusion effect, we can describe it by the following mass transfer around the bubble, assuming a steady state:

$$
\frac{d^{2} c}{d r^{2}}+\frac{2}{r} \frac{d c}{d r}=0
$$

And the boundary conditions are as follows:

$$
r=R_{\mathrm{B}}, \quad c=c_{i} ; \quad r=\infty, \quad c=c_{0} .
$$

And then, we can solve the equation, obtaining concentration distribution as follows:

$$
c=c_{0}-\frac{R_{\mathrm{B}}}{r}\left(c_{0}-c_{i}\right) .
$$




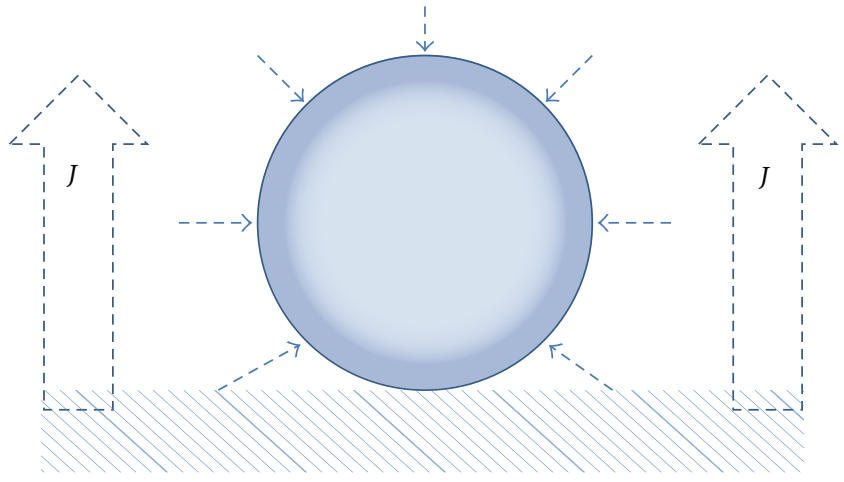

(a) Gas molecules transfer into bubble from gas dissolved in electrolyte

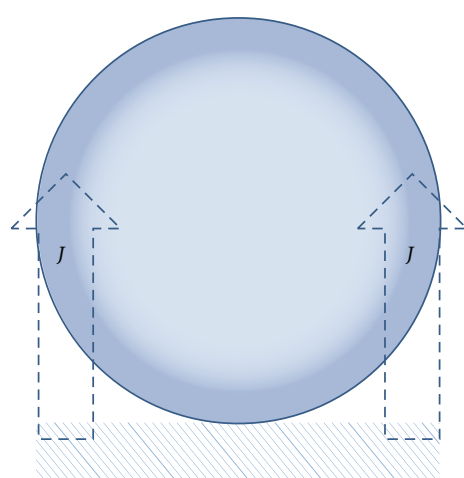

(b) Gas molecules injected into bubble directly from gas reaction produced

FIGURE 6: Bubble growth mechanisms.

So diffusion flow of the gas transferred across the bubble interface can be described as

$$
J_{1}=\left.4 \pi R_{\mathrm{B}}^{2} D_{\mathrm{o}_{2}} \frac{\partial c}{\partial r}\right|_{r=R_{\mathrm{B}}}=4 \pi R_{\mathrm{B}} D_{\mathrm{o}_{2}}\left(c_{0}-c_{i}\right),
$$

where $D_{\mathrm{O}_{2}}$ is the diffusion coefficient of oxygen gas.

For the microconvection effect caused by the bubble interface movement, we refer to the surface renewal theory by Danckwerts [16] described as follows:

$$
J_{2}=4 \pi R_{\mathrm{B}}^{2} \sqrt{S D_{\mathrm{O}_{2}}}\left(c_{0}-c_{i}\right),
$$

where $S$ is ratio of the increasing surface area to the total surface area,

$$
S=\frac{\left(d\left(4 \pi R_{\mathrm{B}}^{2}\right) / d t\right)}{4 \pi R_{\mathrm{B}}^{2}}=\frac{2}{R_{\mathrm{B}}} \frac{d R_{\mathrm{B}}}{d t} .
$$

So the total mass-transfer flow into a bubble is

$$
J=J_{1}+J_{2}=4 \pi R_{\mathrm{B}}^{2} D_{\mathrm{o}_{2}}\left(\frac{1}{R_{\mathrm{B}}}+\sqrt{\frac{2}{R_{\mathrm{B}} D_{\mathrm{o}_{2}}} \frac{d R_{\mathrm{B}}}{d t}}\right)\left(c_{0}-c_{i}\right) .
$$

On the other hand, from the aspect of mass conservation, the net mass-transfer flow is equal to the gas molecules variation in the bubble, which is

$$
J=\frac{d}{d t}\left(\frac{V_{\mathrm{B}}}{V_{\mathrm{mol}}}\right)=\frac{d}{d t}\left(\frac{4 \pi R_{\mathrm{B}}^{3}}{3 V_{\mathrm{m}}}\right) .
$$

And then, we obtain

$$
\frac{d R_{\mathrm{B}}}{d t}=V_{\mathrm{mol}} D_{\mathrm{o}_{2}}\left(c_{0}-c_{i}\right)\left(\frac{1}{R_{\mathrm{B}}}+\sqrt{\frac{2}{R_{\mathrm{B}} D_{\mathrm{o}_{2}}} \frac{d R_{\mathrm{B}}}{d t}}\right) .
$$

Letting $R_{\mathrm{B}}=B t^{0.5}$ according to our experimental results and substituting it to (10), we conclude that

$$
B=\sqrt{D_{\mathrm{o}_{2}}}\left(A+\sqrt{A^{2}+2 A}\right),
$$

where $A=V_{\text {mol }}\left(c_{0}-c_{i}\right)$.
So the bubble radius is

$$
R_{\mathrm{B}}=\left(A+\sqrt{A^{2}+2 A}\right) \sqrt{D_{\mathrm{o}_{2}}}(t)^{1 / 2} .
$$

Equation (12), as the bubble growth model, can describe the diffusion-controlled bubble growth process including diffusion and microconvection effects.

\section{Conclusions}

In this paper, we carried out research on nanorod-array structure surface characteristics and single oxygen bubble growth dynamics. By experimental studies of wettability on the photoelcectrode nanorod-array surface and oxygen bubble growth from anode, we analyzed the interaction affecting the gas-solid-liquid contact behaviors and product transportation mechanism, which is controlled by diffusion due to the concentration gradient of dissolved gases in the aqueous electrolyte and the microconvection caused by the bubble interface movement and we emphasize the importance of marangoni force due to nonhomogeneous gas adsorption at the interface and the capillary force caused by contact behavior with nanorod-array surface structure on bubble growth period. In the end, based on the bubble growth characteristics of $R_{\mathrm{B}}(t) \sim t^{0.5}$ in the experiment, a model describing the product transport mechanism was presented.

\section{Abbreviations}

$R_{\mathrm{B}}: \quad$ Bubble radius, $\mathrm{m}$

$c_{i}$ : Concentration at interface, $\mathrm{mol} / \mathrm{L}$

$c_{0}: \quad$ Concentration in bulk liquid, $\mathrm{mol} / \mathrm{L}$

$D_{\mathrm{O}_{2}}$ : Diffusion coefficient of oxygen gas, $\mathrm{m} / \mathrm{s}$

$J: \quad$ Mass-transfer rate, $\mathrm{mol} / \mathrm{s}$

$V_{\text {mol }}:$ Molar volume, $\mathrm{m}^{3} / \mathrm{mol}$

$V_{\mathrm{B}}$ : Bubble volume, $\mathrm{m}^{3}$.

\section{Conflict of Interests}

The authors declare no conflict of interests. 


\section{Acknowledgments}

The authors gratefully acknowledge the financial support of the National Natural Science Foundation of China (no. 51306147, no. 51236007, no. 51323011, and no. 51102194) and China Postdoctoral Science Foundation (no. 2013M532043). One of the authors (Xiaowei $\mathrm{Hu}$ ) was supported by the "Fundamental Research Funds for the Central Universities." And the authors sincerely thank Dr. J. Su for helpful discussions.

\section{References}

[1] A. B. Murphy, P. R. F. Barnes, L. K. Randeniya et al., "Efficiency of solar water splitting using semiconductor electrodes," International Journal of Hydrogen Energy, vol. 31, no. 14, pp. 19992017, 2006.

[2] J. Turner, G. Sverdrup, M. K. Mann et al., "Renewable hydrogen production," International Journal of Energy Research, vol. 32, no. 5, pp. 379-407, 2008.

[3] M. G. Walter, E. L. Warren, J. R. McKone et al., "Solar water splitting cells," Chemical Reviews, vol. 110, no. 11, pp. 6446-6473, 2010.

[4] G. M. Wang, H. Y. Wang, Y. C. Ling et al., "Hydrogen-treated $\mathrm{TiO}_{2}$ nanowire arrays for photoelectrochemical water splitting," Nano Letters, vol. 11, no. 7, pp. 3026-3033, 2011.

[5] F. E. Osterloh, "Inorganic nanostructures for photoelectrochemical and photocatalytic water splitting," Chemical Society Reviews, vol. 42, no. 6, pp. 2294-2320, 2013.

[6] Z. S. Li, W. J. Luo, M. L. Zhang, J. Y. Feng, and Z. G. Zou, "Photoelectrochemical cells for solar hydrogen production: current state of promising photoelectrodes, methods to improve their properties, and outlook," Energy \& Environmental Science, vol. 6, no. 2, pp. 347-370, 2013.

[7] M. T. Mayer, Y. J. Lin, G. B. Yuan, and D. W. Wang, "Forming heterojunctions at the nanoscale for improved photoelectrochemical water splitting by semiconductor materials: case studies on hematite," Accounts of Chemical Research, vol. 46, no. 7, pp. 1558-1566, 2013.

[8] M. A. Henderson, "The interaction of water with solid surfaces: fundamental aspects revisited," Surface Science Reports, vol. 46, no. 1-8, pp. 1-308, 2002.

[9] L. E. Scriven, "On the dynamics of phase growth," Chemical Engineering Science, vol. 10, no. 1-2, pp. 1-13, 1959.

[10] N. P. Brandon and G. H. Kelsall, "Growth-kinetics of bubbles electrogenerated at microelectrodes," Journal of Applied Electrochemistry, vol. 15, no. 4, pp. 475-484, 1985.

[11] D. Fernandez, M. Martine, A. Meagher, M. E. Mobius, and J. M. D. Coey, "Stabilizing effect of a magnetic field on a gas bubble produced at a microelectrode," Electrochemistry Communications, vol. 18, no. 1, pp. 28-32, 2012.

[12] H. Vogt, "The role of single-phase free-convection in masstransfer at gas evolving electrodes-I. Theoretical," Electrochimica Acta, vol. 38, no. 10, pp. 1421-1426, 1993.

[13] H. Vogt, "The role of single-phase free-convection in mass-transfer at gas evolving electrodes-II. Experimentalverification," Electrochimica Acta, vol. 38, no. 10, pp. 1427-1431, 1993.

[14] J. J. Wei, Y. F. Xue, J. F. Zhao, and J. Li, "Bubble behavior and heat transfer of nucleate pool boiling on micro-pin-finned surface in microgravity," Chinese Physics Letters, vol. 28, no. 1, Article ID 016401, 2011.
[15] C. Yang, T. Dabros, D. Q. Li, J. Czarnecki, and J. H. Masliyah, "Kinetics of microbubble-solid surface interaction and attachment," AIChE Journal, vol. 49, no. 4, pp. 1024-1037, 2003.

[16] P. Danckwerts, "Significance of liquid-film coefficients in gas absorption," Industrial \& Engineering Chemistry, vol. 43, no. 6, pp. 1460-1467, 1951. 

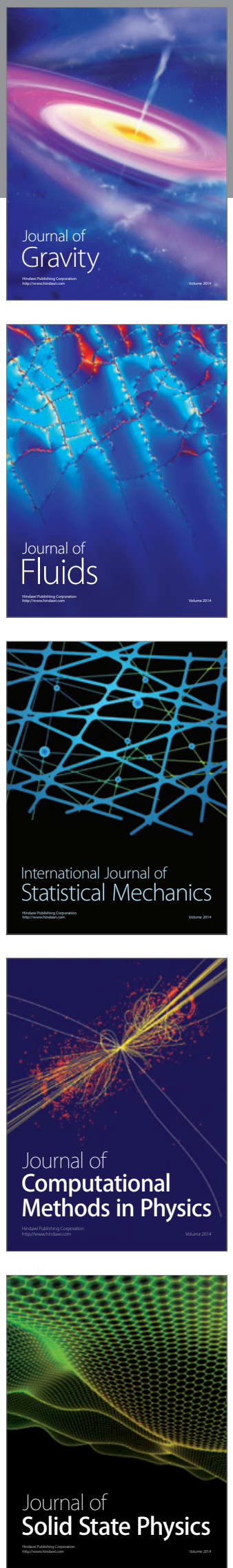

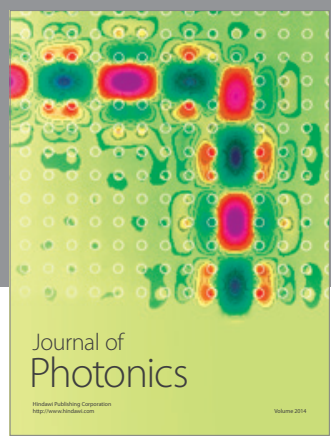

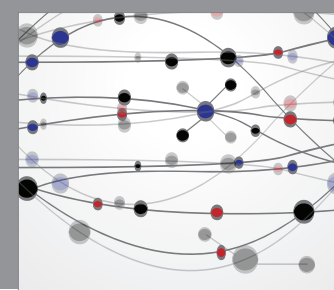

The Scientific World Journal

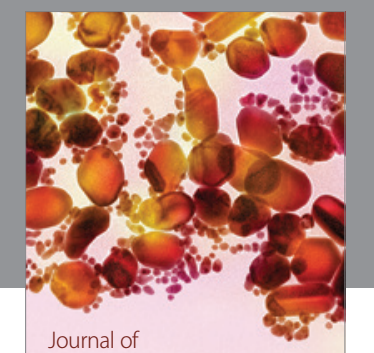

Soft Matter
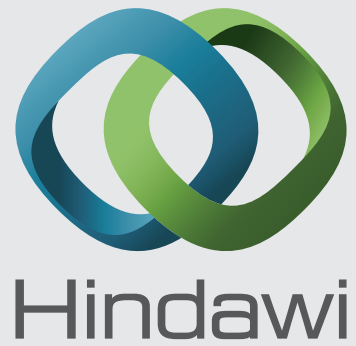

Submit your manuscripts at

http://www.hindawi.com
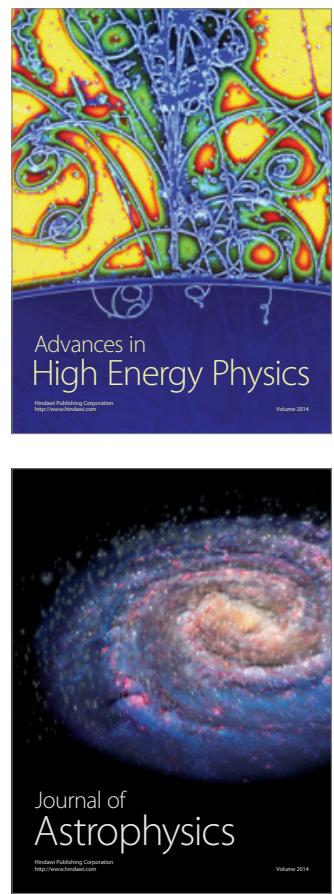
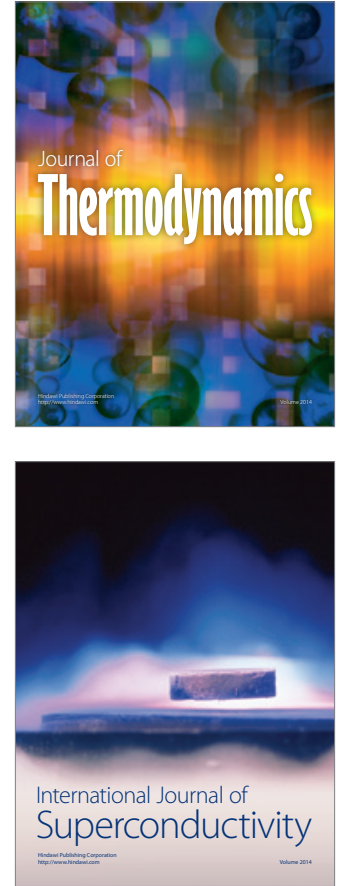
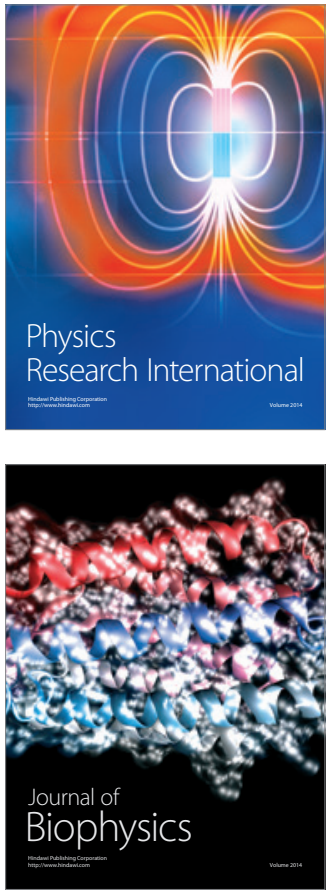
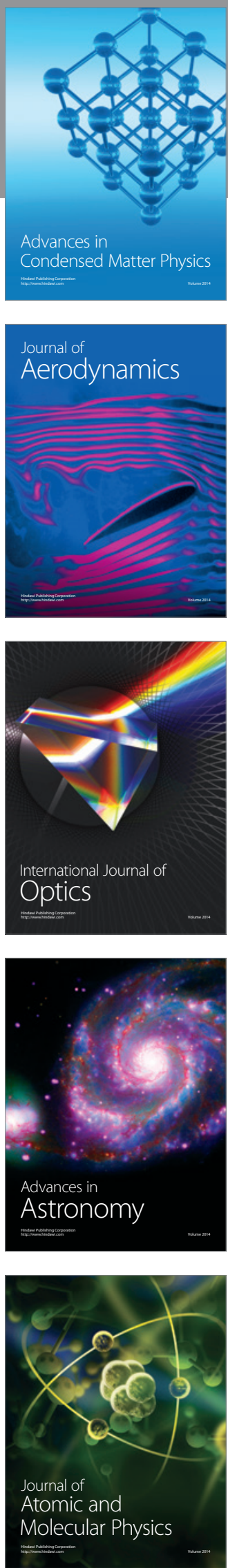